Proceedings of International Mathematical Sciences

ISSN:2717-6355, URL: HTTPS://DERGIPARK.ORG.TR/TR/PUB/PIMS

Volume III Issue 1 (2021), Pages 38-49.

DOI: HTTPS://DOI.ORG/10.47086/PIMS.956201

\title{
DERIVATION OF BLACK-SCHOLES EQUATION USING ITÔ'S LEMMA
}

\author{
BRANDON WASHBURN* AND MEHMET DIK** \\ *BELOIT COLLEGE, BELOIT, WI, UNITED STATES. ORCID NUBMER: \\ 0000-0002-5815-3873 \\ **ROCKFORD UNIVERSITY, ROCKFORD, IL UNITED STATES.
}

\begin{abstract}
The Black-Scholes Equation is arguably the most influential financial equation, as it is an effective example of how to eliminate risk from a financial portfolio by using a hedged position. Hedged positions are used by many firms, mutual funds and finance companies to increase the value of financial assets over time. The derivation of the Black-Scholes equation is often considered difficult to understand and overly complicated, when in reality most confusion arises from misunderstandings in notation or lack of intuition around the mathematical processes involved. This paper aims to take a simple look at the derivation of the Black-Scholes equation as well as the reasoning behind it.
\end{abstract}

\section{INTRODUCTION}

1.1. A Brief History of Black-Scholes Equation. The Black-Scholes Equation is a partial differential equation that gives the theoretical price of a European-style option for a certain security. First derived in 1968 by Fischer Black and Myron Scholes, it was revolutionary in presenting ideas of how to eliminate unpredictable terms from mathematical models.

Their results were published in the Journal of Political Economy in 1973 as an article title "The Pricing of Options and Corporate Liabilities". Fischer Black unfortunately passed away in 1995. Myron Scholes is still alive and would go on to win the Nobel Prize in economics in 1997 along with contributor Robert C. Merton 6] for their work, however Black would be recognized for his contributions by the Nobel Committee. 2]

1.2. A Brief History of Itô/Stochastic Calculus. Keyosi Itô was a Japanese mathematician who pioneered many of the mathematical concepts behind the

2021 Mathematics Subject Classification. 91G15, 91-10.

Key words and phrases. Black-Scholes equation, Financial markets.

(C) 2021 Proceedings of International Mathematical Sciences.

Submitted on 22.06.2021, Accepted on 06.07.2021.

Communicated by İbrahim Canak. 


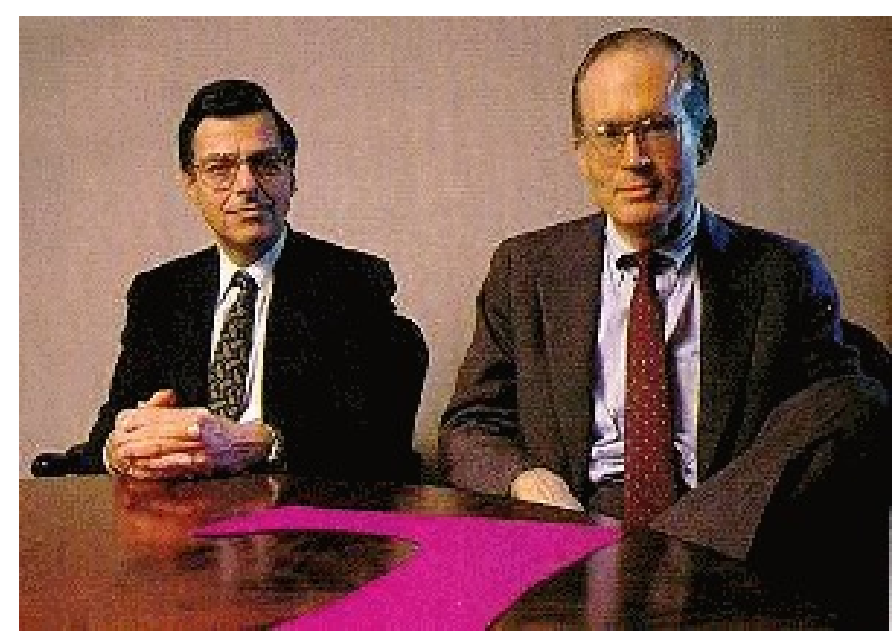

FiguRE 1. Myron Scholes and Fischer Black 8

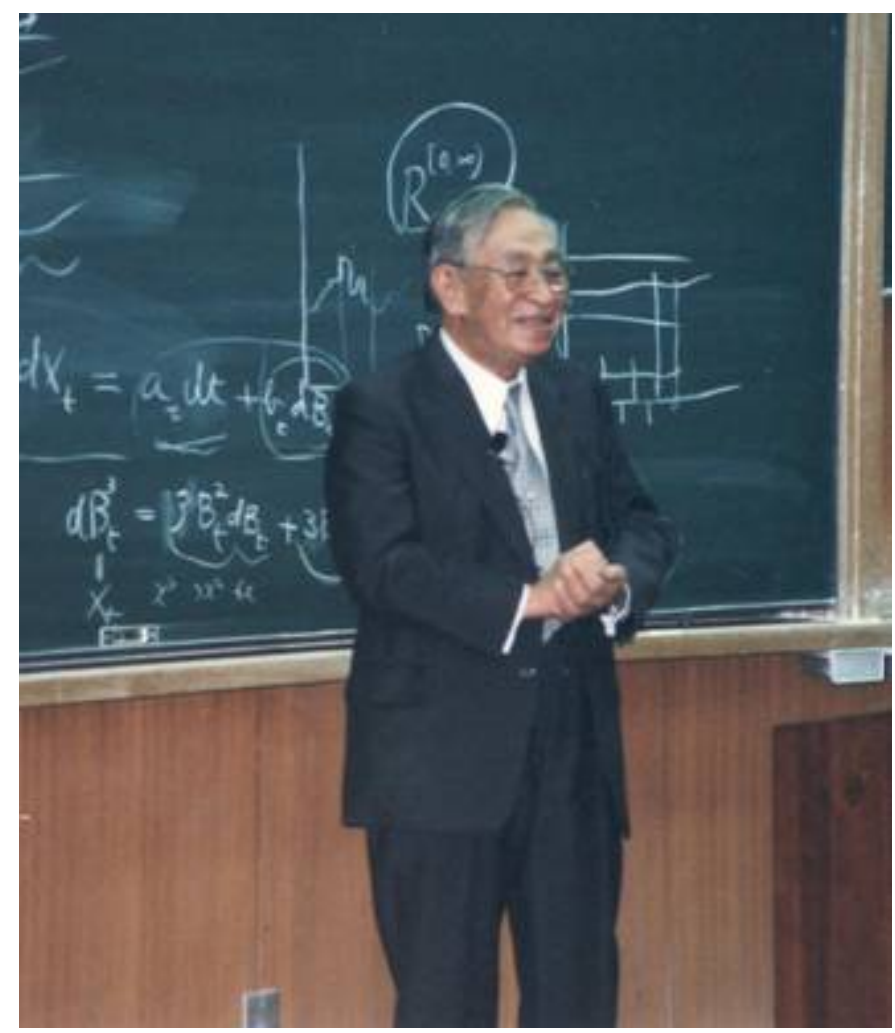

Figure 2. Kiyosi Itô at Kyoto University in 1995[1]

Black-Scholes Equation, the most important of which is Itô's Lemma. His insightful techniques allowed for the creation of his own field of calculus, Itô Calculus. In this 
field of mathematics, he explored stochastic processes, processes that are based in randomness and could not be quantified by normal arithmetic means. As stated by the National Academy of Science, "If one disqualifies the Pythagorean Theorem from contention, it is hard to think of a mathematical result which is better known and more widely applied in the world today than "Ito's Lemma." This result holds the same position in stochastic analysis that Newton's fundamental theorem holds in classical analysis. That is, it is the sine qua non of the subject." His work has implications across other fields such as stochastic control theory in engineering, conformal form theory in physics, modeling of population genetics in biology and more. 1] Kiyosi Itô unfortunately passed away in 2008 at the age of 93.

\section{Intuition of Stochastic Processes}

In this section, we aim to cover the basic intuition behind a stochastic process, enough to understand an Itô Process and Itô's Lemma. We are only going to cover Standard Brownian Motion to understand the theoretical processes behind stochastic calculus. Standard Brownian motion is sometimes referred to as a Wiener Process.

2.1. Basic Definitions of Standard Brownian Motion. There are a few basic definitions that we should cover to begin:

(1) We define Brownian Motion at time $t$ as $B_{t}$

(2) Standard Brownian Motion begins at the origin. Written in our notation, $B_{0}=0$

(3) The difference of Brownian Motion from time $t_{1}$ to time $t_{2}$ can be written as $B_{2}-B_{1}$ where $B_{1}$ is the Brownian Motion at time $t_{1}$ and $B_{2}$ is the Brownian Motion at time $t_{2}$

(4) $B_{2}-B_{1}$ is normally distributed with a $\mu=0$ and a $\sigma=t_{2}-t_{1}$

(5) The derivative of $B_{t}$ can be written as $d B_{t}$ and the integral (without limits of integration) of $d B_{t}$ can be written as $B_{t}$

2.2. Intuition of Brownian Motion. In Figure 3 we can see an example of $f^{\prime}(x)$, a real valued continuous function. There are many things we can do with this function. We are able to integrate $f^{\prime}(x)$ from point $a$ to point $b$, we can take the derivative of $f^{\prime}(x)$ at any point and we can show $f^{\prime}(x)$ is continuous at any point.

Now let's take a look at Brownian Motion. Using computer software, it is possible to simulate 2-dimensional Brownian Motion from one time period to another. In Figure 4, we examine a single simulated Brownian Motion walk with standard parameters from time 0 to time 1 . This motion does not follow typical or predictable paths compared to the path of a function. Here, our sample points fluctuate in an unpredictable and random way, based on the normal distribution. Furthermore, this motion will be different every time we simulate it. In theory, it should be impossible to replicate the same motion more than once[7. This leads us into several problems. We are no longer able to quantify the motion using a function, we cannot prove that the motion is continuous from point $a$ to point $b$, we cannot integrate via Newtonian means with respect to time from point $a$ to point $b$ and we cannot take the derivative with respect to time at any point. 


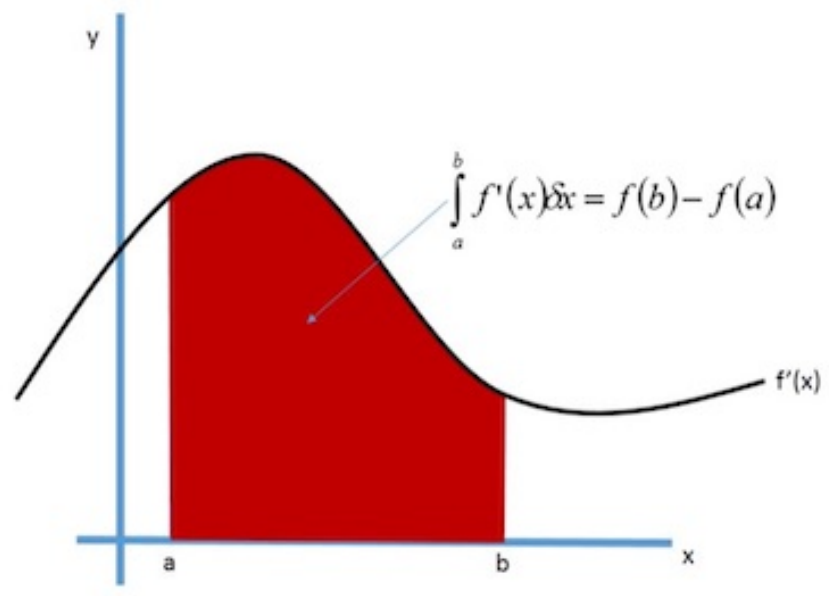

Figure 3. A real valued function $f^{\prime}(x)$ with a smooth curve 3

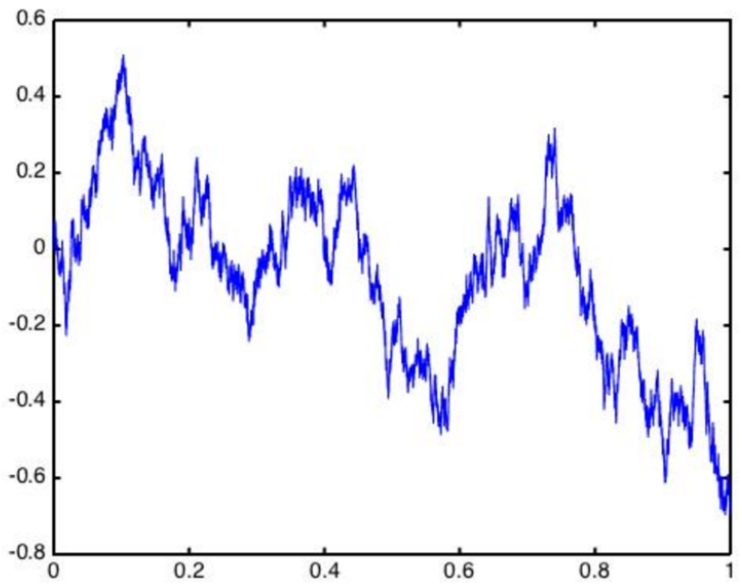

Figure 4. Graph of Standard Brownian Motion from $t=0$ to $t=1[\underline{5}$

An interesting thing to note is that while single simulations of Brownian Motion do not yield any sort of pattern, multiple simulations do. As the number of simulations increases we start to see the emergence of the normal distribution curve, all be it horizontally.

2.3. Derivatives with Respect to Brownian Motion. Intuitively $B_{t}$ is not differentiable, at least not in a normal sense. But if we examine $B_{t}$ in a similar sense to Newtonian calculus, we can arrive at some interesting results. 


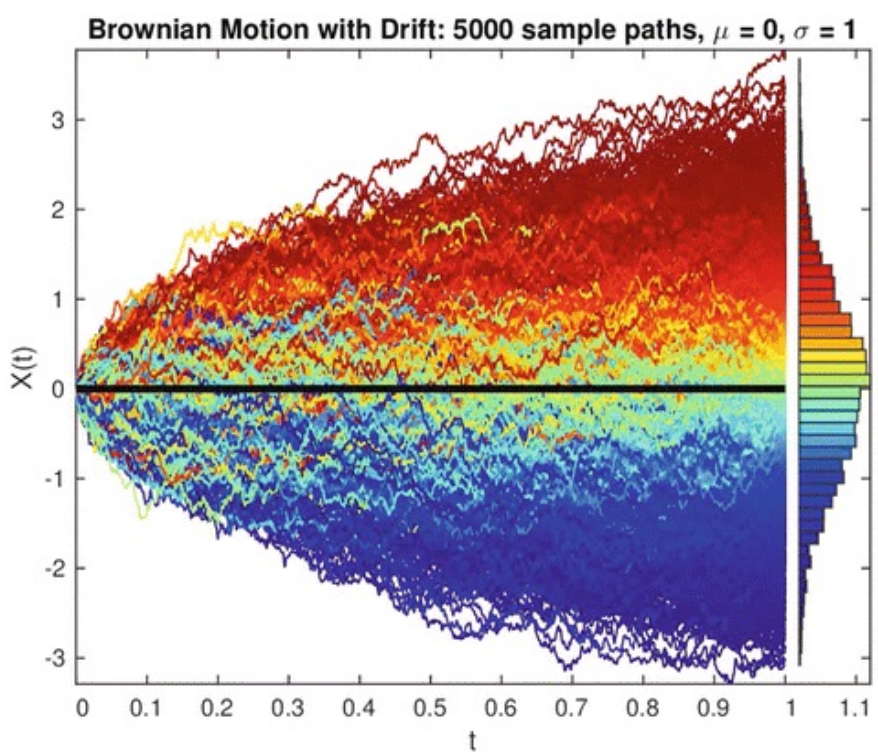

Figure 5. 5000 sample paths of Standard Brownian Motion[7]

In Newtonian calculus, we can take the derivative of a function

$$
g(t)=g(0) e^{r t}
$$

very easily. The derivative is

$$
\frac{d g}{d t}=g(0) e^{r t} * r
$$

Now if we add a Brownian Motion with a scalar beta term to $g(t)$ to create a new equation $s(t)$

$$
s(t)=s(0) e^{r t+\beta B_{t}}
$$

Upon attempting to differentiate $s(t)$ we find

$$
\frac{d s}{d t}=\left[r+\beta \frac{d B_{t}}{d t}\right] s(0) e^{r t+\beta B_{t}}=\left[r+\beta \frac{d B_{t}}{d t}\right] s(t)=r s(t)+\beta s(t) \frac{d B_{t}}{d t}
$$

However, at this time, we have no way of quantifying

$$
\frac{d B_{t}}{d t}
$$

We can further attempt at a separation of variables

Becomes

$$
\frac{d s}{d t}=r s(t)+\beta s(t) \frac{d B_{t}}{d t}
$$

$$
d s=r s(t) d t+\beta s(t) \frac{d B_{t}}{d t} d t
$$

The $d t^{\prime} s$ cancel in the second term to give us

$$
d s=r s(t) d t+\beta s(t) d B_{t}
$$


Once again we have a term we are unfamiliar with

$$
d B_{t}
$$

So as of now, it does not appear we can take the derivative of a process involving a Brownian Motion term. We will return to this discussion later.

2.4. Integration with Respect to Brownian Motion. Since we cannot take the derivative of terms with Brownian Motion, or a stochastic process, let's examine how integration with respect to Brownian Motion theoretically should behave.

2.4.1. Integration of a Constant Function. Let $x(t)=k$ a constant. If we wish to take the integral from 0 to some time $t_{1}$ with respect to Standard Brownian Motion we can write this as

$$
\int_{0}^{t_{1}} x(t) d B_{t}
$$

Evaluating this integral via Newtonian means,

$$
\int_{0}^{t_{1}} x(t) d B_{t}=\int_{0}^{t_{1}} k d B_{t}=k \int_{0}^{t_{1}} d B_{t}=k\left[\left.B_{t}\right|_{0} ^{t_{1}}\right]=k\left[B_{t_{1}}-B_{0}\right]=k B_{t_{1}}
$$

Here we see that integrating a constant with respect to Brownian Motion gives us the constant multiplied by the Brownian Motion at the upper limit of the integral.

2.4.2. Integration of a Step Function. Let $\Phi(t)$ be a step function with $n$ steps. Let $k_{i}$ be the value of the step function at $i=1,2,3, \ldots ., n$ Taking the integral of a step function is effectively taking the the sum of the integral of each constant with respect to Brownian Motion. So we can write

$$
\int_{a}^{b} \phi(t) d B_{t}
$$

as

$$
\sum_{i=1}^{n} \int_{a}^{b} k_{i} d B_{t}
$$

Evaluating this as a sum of all of the integrals of each constant $k_{i}$ we can see

$$
\sum_{i=1}^{n} \int_{a}^{b} k_{i} d B_{t}=\sum_{i=1}^{n} k_{i}\left[B_{b}-B_{a}\right]
$$

and from our definitions we know that

$$
\left[B_{b}-B_{a}\right] \sim \mathcal{N}(0,(b-a))
$$

Leading us to the result that the integral of a function with a term involving Brownian Motion will have a distribution. 
2.4.3. Integration of a Well-Behaved Function. Normally in Newtonian calculus, integration begins with a Riemann sum. If we have a continuous real valued function $f(t)$ and we wish to find the area underneath the curve from $a$ to $b$, the Riemann sum can be written as

$$
\text { Area }=\sum_{a}^{b} f(t) * \Delta t
$$

If we send the length of the partitions of this sum, $\Delta t$, to zero

$$
\lim _{\Delta t \rightarrow 0} \sum_{a}^{b} f(t) * \Delta t=\int_{a}^{b} f(t) d t
$$

The result is our standard Newtonian integration. We can apply this concept to Brownian Motion in a similar way. With a step function, our integral could be defined as

$$
\int_{a}^{b} \phi(t) d B_{t}=\sum_{i=1}^{n} k_{i}\left[B_{b}-B_{a}\right]
$$

Where

$$
\left[B_{b}-B_{a}\right] \sim \mathcal{N}\left(0, K^{2}(b-a)\right)
$$

Effectively if we wanted to evaluate an integral of $f(t)$ with respect to Brownian Motion, we can again partition our function $f(t)$ into sections with widths of $\Delta t$. A well-behaved function evaluated via Riemann sum can be compared to a step function being evaluated by our process in 2.4.2. By the same process as before, if we wish to evaluate

$$
\int_{a}^{b} f(t) d B_{t}
$$

We can approximate it to a step function, and partition $f(t)$ into $\mathrm{n}$ partitions, each with length $\Delta t$. As we take smaller and smaller partitions, we will get a closer and closer approximation of the function. In theory then,

$$
\lim _{\Delta t \rightarrow 0} \int_{a}^{b} f(t) d B_{t} \approx \int_{a}^{b} f(t) d t \sim \mathcal{N}\left(0, \int_{a}^{b}[f(t)]^{2} d t\right)
$$

However, because it is impossible to fully quantify a function to Brownian Motion, this will only give us an approximation of the integral.

2.4.4. Integration of a Random Variable. Let $Y_{t}$ be a random variable defined by:

$$
Y_{t}= \begin{cases}y_{0} & 0<t<t_{1} \\ y_{1} & t_{1}<t<1\end{cases}
$$

Integrating this random variable with respect to Brownian Motion, if $t<t_{1}$ then

$$
\int_{0}^{t} Y_{t} d B_{t}=y_{0} B_{t}
$$

If $t>t_{1}$ then our integral is 


$$
\int_{0}^{t} Y_{t} d B_{t}=y_{0} B_{t_{1}}+y_{1}\left(B_{t}+B_{t_{1}}\right)
$$

Combining these results, out entire integral can be written over our interval $(0,1)$ as

$$
\int_{0}^{1} Y_{t} d B_{t}= \begin{cases}y_{0} B_{t} & 0<t<t_{1} \\ y_{0} B_{t}+y_{1}\left(B_{1}+B_{t}\right) & t_{1}<t<1\end{cases}
$$

And we are able to quantify our integral with respect to Brownian Motion of a random variable.

\section{Itô Calculus[1]}

The ideas and processes of all of these examples highlight a reoccurring theme. That theme is that each process much be broken into a section we can evaluate via Newtonian means and one that must be evaluated by stochastic means. This comes from applying a discrete scenario where we can effectively separate the randomness of our Brownian Motion terms with the certainty of our $t$ terms. This idea of separation is the methodology and thought process behind the Itô Process.

3.1. Itô Process. An Itô Process is an advanced technique that separates a stochastic process $x_{t}$ into a a sum of two integrals, one with respect to time and one with respect to Brownian Motion.

$$
x_{t}=x_{0}+\int_{0}^{t} \sigma_{t} d B_{t}+\int_{0}^{t} \mu_{t} d t
$$

Where $x_{0}$ is a constant term, $\sigma$ is a process contingent on time that can be integrated with respect to Brownian Motion, and $\mu$ is a process contingent on time that can be integrated with respect to time.

3.2. Itô Differential Equation. Taking the "derivative" of the Itô Process gives us the following differential equation:

$$
d x_{t}=\mu_{t} d t+\sigma_{t} d B_{t}
$$

However, this is only an equation, it cannot be evaluated since we cannot take the derivative with respect to Brownian Motion by normal means. This will serve as our derivative of a stochastic process for the sake of making the calculations we intend to do.

3.3. Itô's Lemma Pre-Remarks. Itô's Lemma is an equation that can be compared to the "chain rule" of Newtonian calculus. The formal proof of Itô's Lemma is beyond the the scope of this paper, however, I have included an informal proof using a Taylor power series approximation that provides the contextual intuition behind the formal proof.

3.3.1. Itô's Lemma. If $f\left(t, x_{t}\right)$ is a twice differentiable scalar function, where $x_{t}$ is defined as an Itô Process. Then

$$
d f=\left(\mu_{t} \frac{\partial f}{\partial x}+\frac{\partial f}{\partial t}+\frac{1}{2} \frac{\partial^{2} f}{\partial x^{2}} \sigma_{t}^{2}\right) d t+\frac{\partial f}{\partial x} \sigma_{t} d B_{t}
$$

Itô's Lemma is essential in the derivation of the Black-Scholes Equation. 
3.3.2. Taylor Series Approximation of Itô's Lemma. Let $x_{t}$ be an Itô Process that satisfies our requirements for an Itô Differential Equation.

$$
d x_{t}=\mu_{t} d t+\sigma_{t} d B_{t}
$$

Normally, if $f(t, x)$ is a two-variable and twice differentiable scalar function, we can write its Taylor expansion as

$$
d f=\frac{\partial f}{\partial t} d t+\frac{\partial f}{\partial x} d x+\frac{1}{2} \frac{\partial^{2} f}{\partial x^{2}} d x^{2}
$$

If we equate $x_{t}$ for $x$ and $\mu_{t} d t+\sigma_{t} d B_{t}$ for $d x$, we can rewrite $d f$ as

$$
d f=\frac{\partial f}{\partial t} d t+\frac{\partial f}{\partial x}\left(\mu_{t} d t+\sigma_{t} d B_{t}\right)+\frac{1}{2} \frac{\partial^{2} f}{\partial x^{2}}\left(\mu_{t}^{2} d t+2 \mu_{t} \sigma_{t} d t d B_{t}+\sigma_{t}^{2} d B_{t}^{2}\right)
$$

which means

$$
d f=\frac{\partial f}{\partial t} d t+\frac{\partial f}{\partial x} \mu_{t} d t+\frac{\partial f}{\partial x} \sigma_{t} d B_{t}+\frac{1}{2} \frac{\partial^{2} f}{\partial x^{2}} \mu_{t}^{2} d t+\frac{\partial^{2} f}{\partial x^{2}} \mu_{t} \sigma_{t} d t d B_{t}+\frac{1}{2} \frac{\partial^{2} f}{\partial x^{2}} \sigma_{t}^{2} d B_{t}^{2}
$$

Taking the limit of $d f$ as $d t \rightarrow 0, d t^{2}$ and $d t d B_{t}$ will tend to zero faster than $d t$ and $d B_{t}^{2}$, leading us to the result

$$
\lim _{d t \rightarrow 0} d f=\frac{\partial f}{\partial t} d t+\frac{\partial f}{\partial x} \mu_{t} d t+\frac{\partial f}{\partial x} \sigma_{t} d B_{t}+0+0+\frac{1}{2} \frac{\partial^{2} f}{\partial x^{2}} \sigma_{t}^{2} d B_{t}^{2}
$$

If we replace $d B_{t}^{2}$ with $d t$ (from the quadratic variance of a Wiener process) [9]

$$
d f=\frac{\partial f}{\partial t} d t+\frac{\partial f}{\partial x} \mu_{t} d t+\frac{\partial f}{\partial x} \sigma_{t} d B_{t}+\frac{1}{2} \frac{\partial^{2} f}{\partial x^{2}} \sigma_{t}^{2} d t
$$

Finally factoring out our $d t$ and $d B_{t}$ terms, and separating them

We arrive at Itô's Lemma.

$$
d f=\left(\mu_{t} \frac{\partial f}{\partial x}+\frac{\partial f}{\partial t}+\frac{1}{2} \frac{\partial^{2} f}{\partial x^{2}} \sigma_{t}^{2}\right) d t+\frac{\partial f}{\partial x} \sigma_{t} d B_{t}
$$

\section{BLACK-SCHOLES MOdEL}

The Black-Scholes Model is sometimes referred to the Black-Scholes-Merton Model because of the contributions made by American mathematician Robert C. Merton. He had built upon the ideas presented originally by Myron Scholes and Fischer Black in 1968, adding further mathematical implications and applications to the model. This specific derivation of the equation comes from Hull 4 .

4.1. Assumptions of the Model. The following assumptions are made in the Black-Scholes Model:

(1) The short term interest rate, $r$, is known and constant through time. All securities share this short term interest rate

(2) The stock price follows Brownian Motion and the variance rate of return on the stock is constant.

(3) The parameters $\mu$ and $\sigma$ are contingent on $S$, the stock price

(4) The stock does not pay dividends or other distributions

(5) Short selling is allowed

(6) There are no arbitrage opportunities and all security trading is continuous 
(7) The option has a maturity at time period $t$ (European Option)

4.2. Black-Scholes Equation. If $S$ is a stock price that follows an Itô Process, then the value of an option, $f$, of $S$ is quantified by the following equation:

$$
\frac{\partial f}{\partial t}+r S \frac{\partial f}{\partial S}+\frac{1}{2} \frac{\partial^{2} f}{\partial x^{2}} \sigma^{2} S^{2}=r f
$$

4.3. Derivation. Let $S$ be a stock price that follows an Itô Process. Thus $S$ has a Itô Differential Equation of

$$
d S=\mu S d t+\sigma S d z
$$

Suppose $f$ is a twice differentiable function of the price of a call option or other derivative contingent on $S$. Using Itô's Lemma we can write $d f$ as

$$
d f=\left(\mu S \frac{\partial f}{\partial S}+\frac{\partial f}{\partial t}+\frac{1}{2} \frac{\partial^{2} f}{\partial S^{2}} \sigma^{2} S^{2}\right) d t+\frac{\partial f}{\partial S} \sigma S d z
$$

Notice how $\mu$ and $\sigma$ are no longer contingent on $t$, but are contingent on $S$. (1) and (2) can also be written discretely over a time period $\Delta t$ as

$$
\Delta S=\mu S \Delta+\sigma S \Delta z
$$

and

respectively.

$$
\Delta f=\left(\mu S \frac{\partial f}{\partial S}+\frac{\partial f}{\partial t}+\frac{1}{2} \frac{\partial^{2} f}{\partial S^{2}} \sigma^{2} S^{2}\right) \Delta t+\frac{\partial f}{\partial S} \sigma S \Delta z
$$

(1) Now $S$ and $f$ both follow the same Itô Process. Thus, if we select a portfolio, we can eliminate the Itô Process and effectively price an option. The portfolio we select will consist of short 1 derivative and long $\frac{\delta f}{\delta S}$ shares of stock. It will become clear shortly why we select this portfolio.

(2) We define $\Pi$ as the value of our portfolio. By definition,

$$
\Pi=-f+\frac{\partial f}{\partial S} S
$$

We can also write our the discrete version of this equation over the time interval $\Delta t$ as

$$
\Delta \Pi=-\Delta f+\frac{\partial f}{\partial S} \Delta S
$$

Now we will substitute our (3) and (4) into (6).

$$
\Delta \Pi=-\left[\left(\mu S \frac{\partial f}{\partial S}+\frac{\partial f}{\partial t}+\frac{1}{2} \frac{\partial^{2} f}{\partial S^{2}} \sigma^{2} S^{2}\right) \Delta t+\frac{\partial f}{\partial S} \sigma S \Delta z\right]+\frac{\partial f}{\partial S}[\mu S \Delta+\sigma S \Delta z]
$$

Distributing everything out,

$\Delta \Pi=-\frac{\partial f}{\partial x} \mu S \Delta t-\frac{\partial f}{\partial t} \Delta t-\frac{1}{2} \frac{\partial^{2} f}{\partial S^{2}} \sigma^{2} S^{2} \Delta t-\frac{\partial f}{\partial S} \sigma S \Delta z+\frac{\partial f}{\partial S} \mu S \Delta t+\frac{\partial f}{\partial S} \sigma S \Delta z$

Canceling out like terms,

$$
\Delta \Pi=-\frac{\partial f}{\partial t} \Delta t-\frac{1}{2} \frac{\partial^{2} f}{\partial S^{2}} \sigma^{2} S^{2} \Delta t
$$


Factoring out a $\Delta t$,

$$
\Delta \Pi=\left(-\frac{\partial f}{\partial t}-\frac{1}{2} \frac{\partial^{2} f}{\partial S^{2}} \sigma^{2} S^{2}\right) \Delta t
$$

Without a $\Delta z$ term (stochastic variable), this portfolio is effectively riskless during the time period $\Delta t$. Since there are no arbitrage opportunities, security trading is continuous and all securities share the same short term constant interest rate, our portfolio we have created will earn instantaneous rates of return over the time period $\Delta t$. Thus we can write $\Delta \Pi$ as

$$
\Delta \Pi=r \Pi \Delta t
$$

Now we can substitute our (5) and (7) into (8) and the result is the following equation

$$
\left(-\frac{\partial f}{\partial t}-\frac{1}{2} \frac{\partial^{2} f}{\partial S^{2}} \sigma^{2} S^{2}\right) \Delta t=r\left(-f+\frac{\partial f}{\partial S} S\right) \Delta t
$$

Canceling out a $\Delta t$ on both sides of the equation and multiplying through by -1 ,

$$
\frac{\partial f}{\partial t}-\frac{1}{2} \frac{\partial^{2} f}{\partial S^{2}} \sigma^{2} S^{2}=r\left(f-\frac{\partial f}{\partial S} S\right)
$$

which when rearranged becomes

$$
\frac{\partial f}{\partial t}+r S \frac{\partial f}{\partial S}+\frac{1}{2} \frac{\partial^{2} f}{\partial x^{2}} \sigma^{2} S^{2}=r f
$$

and our derivation is complete.

4.4. Verification of the Model. Now that we have our equation, does it actually work? Let's see through an example. This specific example comes from Hull, John C. (2008) of page 332 .

4.4.1. Example. A forward contract (option) on a non dividend paying stock is a derivative dependent on the stock. We can define the value of the forward contract, $f$, at a general time $t$ in terms of the stock price $S$ as

$$
f=S-K e^{-r(T-t)}
$$

Where $K$ is our delivery price of the option, and the interest on the stock compounds continuously. Now recall our equation (9)

$$
\frac{\partial f}{\partial t}+r S \frac{\partial f}{\partial S}+\frac{1}{2} \frac{\partial^{2} f}{\partial x^{2}} \sigma^{2} S^{2}=r f
$$

We need to find our $\frac{\partial f}{\partial t}, \frac{\partial f}{\partial S}$, and $\frac{\partial^{2} f}{\partial S^{2}}$ in order to verify this equation. Taking the partial derivative of (10) with respect to $t$ we find

$$
\frac{\partial f}{\partial t}=-r K e^{-r(T-t)}
$$

When we take the partial derivative of (10) with respect to $S$ we find

$$
\frac{\partial f}{\partial S}=1
$$

and taking the second partial of this we find 


$$
\frac{\partial^{2} f}{\partial S^{2}}=0
$$

When we substitute these results into the left side of (9) the equation becomes

$$
-r K e^{-r(T-t)}+r S
$$

Factoring out an $r$ our result is

$$
r\left(S-K e^{-r(T-t)}\right)
$$

We can clearly see that equation (11) is simply $r f$. Thus our equation (9), the Black-Scholes Equation, holds.

\section{REFERENCES}

[1] Kiyosi Itô, RIMS (1994).

[2] F. Black, and M. Scholes, The pricing of options and corporate liabilities, World Scientific (2019) 3-21.

[3] T. Habb, What in the world will I ever use this for? Integration, Environmental Economics (2014).

[4] J.C. Hull, Options futures and other derivatives, Pearson Education India (2003).

[5] D. Khoshnevisan, and Y. Xiao, Stochastic analysis and related topics, Springer (2017) 179206.

[6] R.C. Merton, Theory of rational option pricing, The Bell Journal of Economics and Management Science (1973) 141-183.

[7] A. Petters, and X. Dong, Stochastic calculus and geometric brownian motion mode, An Intro. Math. Finance. App. Springer (2016) 253-327.

[8] K. Rubash, Myron Scholes and Fischer Black, Bradley University.

[9] H. Stark, and J.W. Woods, Probability, random processes, and estimation theory for engineers, Prentice-Hall, Inc. (1986).

BRANDON WASHBURN,

5028 S 36th St, Greenfield, WI 53221, Phone: 414-333-7564 Orcid nubmer: 0000-0002$5815-3873$

Email address: 17bwashburn@gmail.com

Mehmet Dik,

5050 E State St, Rockford, IL Phone: 815-226-4135 OrChid Number: 0000-0003-0643-2771

Email address: mdik@rockford.edu 